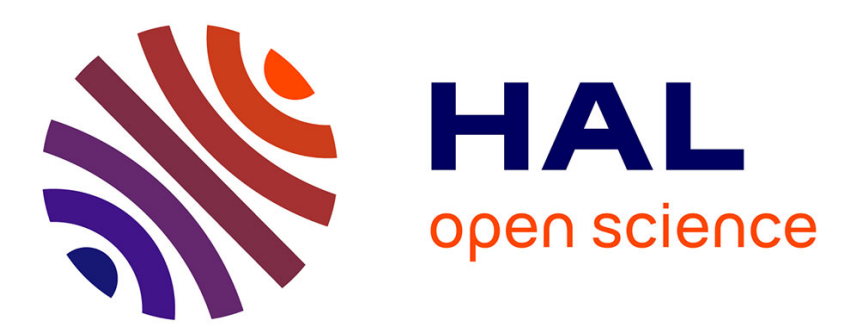

\title{
The AfriSAR Campaign: Tomographic Analysis with Phase-Screen Correction for P-Band Acquisitions
}

Valentine Wasik, Pascale C. Dubois-Fernandez, Cédric Taillandier, Sassan S. Saatchi

\section{- To cite this version:}

Valentine Wasik, Pascale C. Dubois-Fernandez, Cédric Taillandier, Sassan S. Saatchi. The AfriSAR Campaign: Tomographic Analysis with Phase-Screen Correction for P-Band Acquisitions. IEEE Journal of Selected Topics in Applied Earth Observations and Remote Sensing, 2018, pp.1-13. 10.1109/JSTARS.2018.2831441 . hal-01829441

\section{HAL Id: hal-01829441 https://hal.science/hal-01829441}

Submitted on 4 Jul 2018

HAL is a multi-disciplinary open access archive for the deposit and dissemination of scientific research documents, whether they are published or not. The documents may come from teaching and research institutions in France or abroad, or from public or private research centers.
L'archive ouverte pluridisciplinaire HAL, est destinée au dépôt et à la diffusion de documents scientifiques de niveau recherche, publiés ou non, émanant des établissements d'enseignement et de recherche français ou étrangers, des laboratoires publics ou privés. 


\title{
The AfriSAR campaign: Tomographic Analysis with Phase Screens Correction for P-bands Acquisitions
}

\author{
Valentine Wasik, Pascale C. Dubois-Fernandez, Cédric Taillandier and Sassan Saatchi
}

\begin{abstract}
The next European Space Agency (ESA) Earth Explorer mission BIOMASS will acquire Synthetic Aperture Radar (SAR) data to help characterizing carbon fluxes in densely vegetated areas. The ESA-sponsored AfriSAR campaign was designed to collect data from African tropical forests in order to support the future BIOMASS mission. It was conducted in two parts over the tropical forests of Gabon, by ONERA in July 2015 and by DLR in Febuary 2016. This paper addresses the potential of tomographic SAR for retrieving vegetation parameters from the multibaseline P-band airborne data acquired by ONERA over the forest of La Lopé. It is shown that a correction of phase disturbances (phase screens) is necessary before tomographic analysis. Under the hypothesis of phase screens resulting only from inaccurancies in the platform motion, a correction procedure based on recent works from Tebaldini $e t$ al. is detailed and applied. The tomographic profiles after correction are shown to present good correspondances with the available LIDAR data.
\end{abstract}

\section{INTRODUCTION}

Modelling carbon cycle on Earth and understanding its implication in climate change have become crucial environmental challenges. In particular, tropical forests play an essential role in this mecanism. However, the quantity of carbon stocked by the trees and the fluxes between vegetation and atmosphere. Moreover, tropical forests represents a landscape in permanent evolution due to deforestation or reforestation of degradated areas. A constant monitoring of these forests is necessary in order to characterize the impact of their dynamics in carbon fluxes. In this context, the European Spatial Agency (ESA) BIOMASS mission has been selected to be the next Earth Explorer Core mission: in near future, global observation and monitoring of forests biomass will be ensured by the BIOMASS satellite with a Synthetic Aperture Radar (SAR) system [1]. BIOMASS will be implemented as a P-band SAR system to allow a better penetration in the canopy cover. The system will function in polarisation mode with a repeat-pass interferometric orbit and a revisit time of 3-4 days, so that polarimetric and interferometric methods can be implement to analyze the forests parameters.

The ESA-sponsored AfriSAR airborne campaign has been specifically designed to collect data in the context of the BIOMASS mission [2]. This campaign have been conducted in two parts over the tropical forests of Gabon (Africa), firstly in July 2015 with ONERA airborne system SETHI, and secondly in Febuary 2016 with DLR airborne systeme F-SAR. The data

V. Wasik and P. C. Dubois-Fernandez are with the Department of Electromagnetism and Radar, Office National d'Études et de Recherches Aerospatiales, 13661 Salon-de-Provence, France.

C. Taillandier is with TOTAL, Centre Scientifique et Technique Jean-Féger, 64000 Pau, France.

S. Saatchi is with the Radar Science and Engineering Section, Jet Propulsion Laboratory, California Institute of Technology, Pasadena, CA 91109 USA. collection strategy was constructed in order to assess specific questions such as the verification of BIOMASS geophysical inversion models over African tropical forests with biomass ranging from 100t/ha to 600t/ha, the assessment of the P-band SAR imaging for deforestation and degradation monitoring, or the analysis of the impact of seasonal changes in the estimation procedure.

On ONERA side, the AfriSAR campaign was also part of an on-going project for TOTAL, which one of the objectives is to study the potential of SAR imaging for estimating the undercanopy topography in order to understand the geomorphology of terrains.

Among the image processing techniques considered for the BIOMASS mission, SAR tomography is interesting for the retrieval of vegetation and soil parameters, such as forests structure, vegetation height, ground elevation or aboveground biomass, and has been the subject of a dynamic scientific research this last two decades [3], [4], [5], [6], [7], [8]. This method is based on the use of multibaseline data acquired by parallel flights at different altitudes over a same scene, which give access to a 3-D resolution capability. However, phase disturbances originating from uncertainities in trajectories of the plateform transporting the SAR system, or from other perturbations on the propagation path, make the multibaseline data not directly exploitable for tomography analysis. These phase disturbances (also called phase screens) need to be estimated and eliminated from the data to ensure a good interpretation of the tomographic results.

In this article, we will focus on the phase screen correction of the P-band multibaseline data acquired over La Lopé National Park by ONERA during the July 2015 AfriSAR campaign. We will then propose a first tomographic analysis of several regions of this forest. In section II, an overview of the site of La Lopé and on the radar acquisitions configuration will be presented. The basic principle of SAR tomography along with the phase screens problematic will be reminded in section III. A method for phase screen correction largely inspired by the work of Tebaldini et al. in [9] is proposed in section IV. In section $\mathrm{V}$, the tomographic results from the P-band ONERA acquisitions over La Lopé are presented and compared to the available LIDAR data.

\section{THE AFRISAR CAMPAIGN}

\section{A. The AfriSAR sites}

The ESA-sponsored AfriSAR campaign has been carried out over the tropical forest of Gabon in Africa by ONERA (in July 2015) and by DLR (in Febuary 2016). Four sites presenting different forest structures have been selected: La 
Lopé, Mabounie, Mondah and Rabi, located respectively at $250 \mathrm{~km}, 180 \mathrm{~km}, 25 \mathrm{~km}$ and $260 \mathrm{~km}$ from the Libreville airport where the calibration site was deployed. In this section, we will focus on the presentation of La Lopé, which is the main acquisition site.

La Lopé is a National Park, part of the World Heritage List of UNESCO since 2007, covering $4970 \mathrm{~km}^{2}$. The park is routinely monitored by the National Park Agency of Gabon (ANPN), which makes it a well documented site perfect for scientific research. It is a patchy landscape composed of large savanna areas and dense tropical forests, with a biomass ranging approximatively from close to $0 \mathrm{t} / \mathrm{ha}$ to $600 \mathrm{t} / \mathrm{ha}$. The topography is strongly marked, with hills presenting large slopes.

\section{B. Radar acquisitions configuration}

The airborne SAR system used for the AfriSAR ONERA campaign is the SETHI system, developed by ONERA, onboard a Falcon 20. Two pods located under the aircraft wings carry the optronics and the microwaves sensors (ranging from $\mathrm{P}$ to $\mathrm{X}$ band). In order to facilitate comparison between the data acquired in July 2015 and in Febuary 2016, ONERA and DLR have defined a common radar configuration for P-band imaging for both their radar systems. The characteristics of the acquisition geometry and the selected P-band waveform are indicated on table I.

\begin{tabular}{cc}
\hline \multicolumn{2}{c}{ Geometry } \\
\hline Flight ground altitude \\
Aircraft speed & $20000 \mathrm{ft}$ or $6096 \mathrm{~m}$ \\
Rodar setting & $100-150 \mathrm{~m} / \mathrm{s}$ \\
\hline Pode / Side & P-band / Left-looking \\
Pulse duration & $30 \mu \mathrm{s}$ \\
Sampling frequency & $500 \mathrm{MHz}$ \\
Frequency range* / Bandwidth & $410-460 \mathrm{MHz} / 50 \mathrm{MHz}$ \\
Polarimetric mode* & Full $(H \mathrm{H}, \mathrm{HV}, \mathrm{VH}, \mathrm{VV})$ \\
Effective PRF & $1250 \mathrm{~Hz}$ \\
Transmitted power & $500 \mathrm{~W}$ \\
Antenna beamwidth (site / azimuth) & $100^{\circ} / 60^{\circ}$ \\
Range of incidence angle* & $25-55^{\circ}$ \\
Effective swath $^{*}$ SLC images \\
\hline
\end{tabular}

TABLE I

DESCRIPTION OF THE SETHI SYSTEM CONFIGURATION AND THE WAVEFORM FOR AFRISAR P-BAND ACQUISITIONS. THE SUPERSCRIPT * INDICATES THE PARAMETERS THAT ARE IDENTICAL BETWEEN THE SETHI AND THE F-SAR SYSTEMS.

For tomographic analysis purpose, multibaseline SAR acquisitions have been achieved over La Lopé. The multibaseline data set is composed by images acquired with identical ground tracks and at 10 different altitudes over the same scene (see table II). The altitude of each track is indicated with respect to the track 1 (flight height at $6096 \mathrm{~m}$ above the ground).

The full polarimetric single look complex (SLC) images were then processed by ONERA. Each slave SLC image of the multibaseline data set has been projected into the geometry of the chosen master SLC (corresponding to the track 10 of table II). The data have also been phase calibrated using the

\begin{tabular}{cc}
\hline Track & Relative altitude \\
\hline 1 & $0 \mathrm{~m}$ \\
2 & $-80 \mathrm{~m}$ \\
3 & $-60 \mathrm{~m}$ \\
4 & $-20 \mathrm{~m}$ \\
5 & $+10 \mathrm{~m}$ \\
6 & $+40 \mathrm{~m}$ \\
7 & $+60 \mathrm{~m}$ \\
8 & $+80 \mathrm{~m}$ \\
9 & $-80 \mathrm{~m}$ \\
10 (Master) & $+10 \mathrm{~m}$ \\
\hline
\end{tabular}

TABLE II

CONFIGURATION OF THE MULTIBASELINE ACQUISITIONS OVER LA LOPÉ. THE ALTITUDES ARE INDICATED WITH RESPECT TO TRACK 1.

TanDEM-X Digital Elevation Model (DEM) provided by DLR and information about the flights trajectories from the ONERA Inertial Motion Unit (IMU) located onboard. The pixel size of the obtained SLC images are indicated in table I. A colored polarimetric composition of the master SLC image is shown on Figure 1.

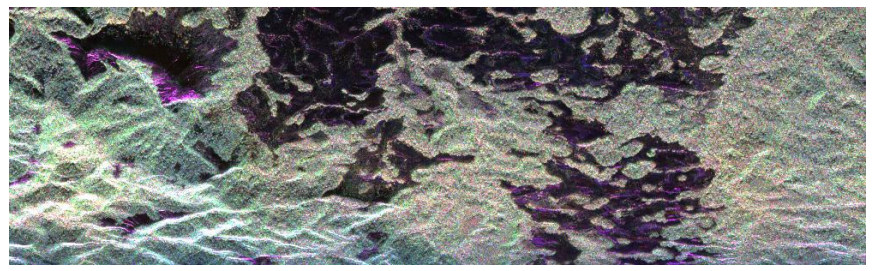

Fig. 1. Colored polarimetric composition (R: $H H, \mathrm{G}: H V, \mathrm{~B}: V V$ ) of the master SLC image from the multibaseline P-band data acquired over La Lopé by ONERA.

\section{Available LIDAR data}

Two LIDAR data sets have been acquired over the area of La Lopé with different instruments.

The first set, which we will refer to as the Small Footprint LIDAR (SFL) data set, was collected by Sassan Saatchi's team (JPL) using a Riegl VQ480U sensor mounted on a Gabonese helicopter model EC 135 in July 2015 with a variable point density and footprint diameter of about $10 \mathrm{~cm}$. The data were collected by a Helimap Systems, a Swiss company providing services in LIDAR and digital photogrammetry under a subcontract from University of California, Los Angeles. The data were pre-processed to remove any artefacts of helicopter motions to provide a more uniform point density of about 10 points per $\mathrm{m}^{2}$ for vegetation characterization. In this study, i) Digital Terrain model (DTM), ii) Canopy Height Model (CHM) and other canopy metrics derived from CHM or point clouds were computed based on the following steps. First, ground returns were classified using Progressive Triangulated Irregular Network (TIN) densification algorithm, and 1m DTM was created [10]. Based on the DTM, slope (\%) maps were computed. Second, SF DR LIDAR-derived data were height aboveground normalized by removing the ground elevation and $1 \mathrm{~m}$ CHM was then computed. The DTM and CHM data were developed in raster format at $1 \mathrm{~m}$ posting characterizing the forest structure an area of approximately $15 \mathrm{~km}^{2}$ of old growth, successional forests, and savanna vegetation of the 
northern Lope National Park.

The obtained CHM and DTM are presented on Figure 2 in the geometry of the SLC radar images acquired by ONERA. Even though the acquisitions of the SFL data were done on a large area of La Lopé, the coverages between the SFL and the SLC images do not match perfectly, which explains the white zones with no data in Figure 2. The large savanna areas with low vegetation are well visible in the CHM representation of Figure 2(a), the tree height of the plots of tropical forest can sometimes exceed $40 \mathrm{~m}$. The hilly topography is also notable on the DTM of Figure 2(b): the available LIDAR data show a culminant point at $520 \mathrm{~m}$ in the radar swath with a $300 \mathrm{~m}$ ground height variation in the radar swath over La Lopé.

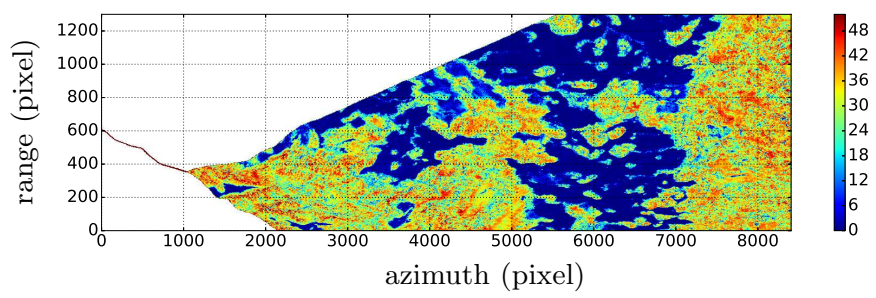

(a) $\mathrm{CHM}(m)$

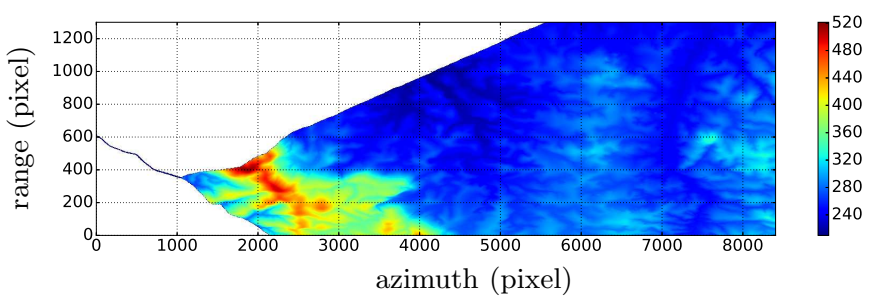

(b) DTM $(m)$

Fig. 2. Canopy Height Model (CHM) and Digital Terrain Model (DTM) in meters derived from the SFL data and projected into the geometry of the SLC images acquired by ONERA.

The second set, referenced as the LVIS data set in the following, was acquired with NASA's airborne Land Vegetation Ice Sensor (LVIS) during March 2016 in the frame of an international AfriSAR cooperation. This instrument is a full-waveform LIDAR system that has shown its potential for retrieving surface topography and vegetation structure (see [11] and [12] for more details).

In section $\mathrm{V}$, we will compare our tomographic results with the two LIDAR data sets previously cited: SFL data and LVIS data.

\section{Regions of Interest for La Lopé}

In situ field plot measurements in La Lopé were carried on by the University College of London (UCL) and the Centre d'Etudes Spatiales de la BIOsphere (CESBIO), between January and August 2016. In total, 12 plots have been explored, from savannas to densely forested areas. In table III, we present the characteristics of 4 of these Regions of Interest (ROIs) that will be the subject of the tomographic analysis of section V. The maximal and minimal values from the CHM SFL data are indicated, as well as the size of the regions in pixels for the P-band SLC images. The SAV2 ROI is a savanna region with low vegetation. The ROIs COL1 and COL3 are colonizing forests located at savannas borders, at different growing states. The $\mathrm{OKO} 2$ plot represents a quasi mono-specy forest of tall Okoumé trees.

\begin{tabular}{ccccc}
\hline ROI & Type & $\begin{array}{c}\text { CHM min } \\
(m)\end{array}$ & $\begin{array}{c}\text { CHM max } \\
(m)\end{array}$ & $\begin{array}{c}N \\
\text { (pixels) }\end{array}$ \\
\hline SAV2 & $\begin{array}{c}\text { Savanna } \\
\text { Colonizing forest } \\
\text { (Intermediate) }\end{array}$ & 0.01 & 3.2 & 1520 \\
COL1 & 6.91 & 43.1 & 640 \\
COL3 & $\begin{array}{c}\text { Colonizing forest } \\
\text { (Young) }\end{array}$ & 0.92 & 17.02 & 595 \\
OKO2 & Okoumés forest & 25.97 & 37.14 & 1944 \\
\hline
\end{tabular}

TABLE III

REGIONS OF INTEREST (ROI) FOR LA LOPÉ. THE SIZE $N$ OF THE REGION IS INDICATED FOR THE P-BAND ACQUISITIONS.

\section{SAR TOMOGRAPHY BACKGROUND}

\section{A. General background}

In the multibaseline airborne case, acquisitions are done from different altitudes of the plateform transporting the radar antenna. Consequently, the scene is imaged with slightly different incidence angles, which gives access to a vertical resolution. As described in the previous section, the SETHI airborne system was used in full polarimetric mode for the AfriSAR campaign, which means that the scenes were alternatively illuminated with two transmit polarizations: horizontal $(H)$ and vertical $(V)$. The backscattered signal is then received in the horizontal and vertical direction. We note $s=\left[s_{1}, \ldots, s_{P}\right]^{T}$ the multibaseline signal, where $s_{p}(p=1, . ., P)$ is the complex backscattered signal received by the plateform $p$ for a polarization channel $X Y$ with $X$ standing for the emitted polarization and $Y$ for the direction of reception $(X, Y=H, V)$. For the sake of simplicity, the $X Y$ dependance is not indicated in the notation of $s$, but one has to keep in mind that this definition stands for one chosen polarization channel. In presence of fully developped speckle [14], $s$ is assumed to be a random vector following a complex circular multivariate Gaussian law with zero-mean, entirely characterized by its covariance matrix $R$ :

$$
\boldsymbol{R}=\left\langle s s^{\dagger}\right\rangle,
$$

where \langle\rangle stands for the statistical average and $\dagger$ is the complex conjugate operator.

For a point target at altitude $z_{g}$, assuming that the distance between the antennas $p$ and $q$ is much lower than the distance to the illuminated target, the complex coefficients $s_{p}$ and $s_{q}$ differ only by their phases:

$$
s_{q}=s_{p} e^{j\left(\varphi_{p}-\varphi_{q}\right)} .
$$

When no perturbation is present on the propagating path, and after flat-earth pre-processing, the phase difference can be linked to the altitude $z_{g}$ of the surface [13]:

$$
\varphi_{p}-\varphi_{q}=k_{z}^{p, q} z_{g},
$$

where

$$
k_{z}^{p, q}=\frac{4 \pi}{\lambda R} \frac{B_{\perp}^{p, q}}{\sin \theta},
$$

is the vertical wavenumber with $\lambda$ the wavelength, $\theta$ the incident angle, $R$ the distance to the point target and $B_{\perp}^{p, q}$ the 
orthogonal projection of the distance separating the antennas $p$ and $q$, also called orthogonal baseline.

For a volumetric target such as vegetated areas, the scatterers are distributed between the ground at altitude $z_{g}$ and the top of canopy at altitude $z_{g}+h_{v}$. Considering that the volume is constituted by statiscally independant continuous layers, the expected value of the backscattered power received for antenna $p$ can be characterized by [15]:

$$
\left\langle\left|s_{p}\right|^{2}\right\rangle=\int_{z_{g}}^{z_{g}+h_{v}} S(z) d z
$$

where $S(z)=\left\langle|P(z)|^{2}\right\rangle$ refers to the backscattered power of the scatterers at altitude $z$ and $P(z)$ is the complex reflectivity of the medium [16] taking into account the attenuation through the vegetation. From Eqs.(2) and (3), the coefficients of the covariance matrix can then be written [15], [16]:

$$
[\boldsymbol{R}]_{p, q}=\left\langle s_{p} s_{q}^{*}\right\rangle=\int_{z_{g}}^{z_{g}+h_{v}} S(z) e^{-j k_{z}^{p, q} z} d z .
$$

Tomographic techniques consist in retrieving the backscattered power $S(z)$ from the multibaseline acquisitions, in order to obtain a representation of the vertical profile of the vegetated areas. In this paper, only spectral estimators applied on single polarization channels $X Y$ will be considered. Full polarization tomographic estimators combining all the polarization channels such as in [6] are not mentionned, although they present an interesting perspective to this work.

\section{B. Capon spectral estimator}

The Capon spectral estimator is a conventional nonparametric method in tomographic analysis, that allows one to obtain a continuous vertical profile of the vegetation without any knowledge on the statistical properties of the data [17], [4]. The Capon estimated vertical profile $\widehat{S}_{C}(z)$ is retrieved from the covariance matrix of the data [4]:

$$
\widehat{S}_{C}(z)=\frac{1}{\boldsymbol{a}(z)^{\dagger} \widehat{\boldsymbol{R}}^{-1} \boldsymbol{a}(z)}
$$

where $\boldsymbol{a}(z)$ is the steering vector containing the interferometric information for a scatter at altitude $z$ for all the baselines relative to a master track $M$ :

$$
\boldsymbol{a}(z)=\left[e^{j k_{z}^{M, 1} z}, e^{j k_{z}^{M, 2} z}, \ldots, e^{j k_{z}^{M, P} z}\right]^{T}
$$

and where $\widehat{\boldsymbol{R}}$ is the maximum likelihood estimation of the covariance matrix:

$$
\widehat{\boldsymbol{R}}=\frac{1}{N} \sum_{n=1}^{N} \boldsymbol{s}_{n} \boldsymbol{s}_{n}^{\dagger}
$$

with $N$ the number of independant measures. Let's note that since the inverse of the estimated covariance matrix is used, the Capon estimator requires $\widehat{\boldsymbol{R}}$ to be well conditioned.

\section{Phase screens}

The previous paragraph described the theoretical model for tomographic analysis assuming no perturbations on the propagating path. However, the radar images are oftenly perturbed by space-varying residual phases (phase screens) which can originate from uncertainities in the position of the antennas [18] or from atmospheric perturbations [19]. For point target, it can be represented by an additional term in the phase $\phi_{p}$ the $s_{p}$ coefficient of the multibaseline signal:

$$
\phi_{p}=\varphi_{p}+\alpha_{p}
$$

where $\alpha_{p}$ the the space-varying phase screen for the image $p$. For a point target of elevation $z_{g}$, the interferometric phase between images $p$ and $q$ can then be written:

$$
\phi_{p, q}=\phi_{p}-\phi_{q}=k_{z}^{p, q} z_{g}+\alpha_{p}-\alpha_{q} .
$$

These phase screens can have important consequences on the tomographic estimation, by emphasing side lobes, defocusing principal lobes or causing other artefacts [15] that make the tomographic profile not directly interpretable without phase screen correction.

An important pre-processing step is then to estimate the $P$ phase screens and to correct the multibaseline data consequently before applying tomographic estimators. This problem of phase screen compensation has been widely explored in the litterature. Some techniques are based on the use of particularly stable point in the images, such as "Permanent Scatterers" [20], [15]. However, this technique has shown its limits for densely forested areas, due to the decorrelation caused by the vegetation. Other approaches propose a separation of the contribution of the ground and of the vegetation to isolate and compensate the ground phase [21], [5]. More recently, Tebaldini et al. proposed a method based on the double estimation of the target position and of the airborne plateform position errors responsible for the phase screens [9]. The method we choose to apply on the La Lopé data is largely inspired by the work of Tebaldini et al. in [9] and will be described in the following section.

\section{Phase SCREENS ESTIMATION AND DATA CORRECTION}

\section{A. Phase Linking algorithm}

As explained in [9], it is important for tomographic analysis to work on data for which the interferometric phases can be expressed as the form given by (11). It is the case for point-like target such as bare surfaces, but for volumetric targets such as forest, it is difficult to link the interferometric phase to a geometrical height of the vegetation due to the loss of coherency between baselines. For this reason, an algorithm called "Phase Linking" has been developed in [22], [9] in order to estimate phases that can be used to model volumetric targets as point-like targets. The general idea is to construct an ensemble of phases $\left\{\phi_{p}^{(\ell)}\right\}_{p=1 . . P}$ using all the available interferograms with a weighting coefficient $w_{n, m}$ that privileges the smallest baselines (i.e. the nearest antennas), for 
which the interferometric coherence is stronger:

$$
\left\{\widehat{\phi}_{p}^{(\ell)}\right\}_{p=1 . . P}=\operatorname{argmax}\left[\sum_{n, m} w_{n, m}\left\langle s_{n} s_{m}^{*}\right\rangle e^{j\left(\widehat{\phi}_{n}^{(\ell)}-\widehat{\phi}_{m}^{(\ell)}\right)}\right],
$$

with $n, m=1 . . P$ and

$$
w_{n, m}=\frac{\left|\left\langle s_{n} s_{m}^{*}\right\rangle\right|}{\left\langle s_{n} s_{n}^{*}\right\rangle\left\langle s_{m} s_{m}^{*}\right\rangle} .
$$

The ensemble average is calculated using windows of $33 \times 33$ pixels on the data. The algorithm of Eq.(12) is an iterative procedure that may depend on the considered initialisation. In this paper, the set of $P$ initial phases $\left\{\widehat{\phi}_{p}^{0}\right\}_{p=1 . . P}$ are chosen to be the circular means of the interferometric phases for all the baselines relatively to the image $p$ :

$$
\widehat{\phi}_{p}^{0}=f\left[\arg \left(\sum_{k=1}^{P} \exp \left(j \beta_{p}^{(k)}\right)\right)\right],
$$

with $\beta_{p}^{(k)}=\arg \left(\left\langle s_{p} s_{k}^{*}\right\rangle\right)$, with $\arg (a)$ is the argument of the complex number $a$, and where $f$ is a smoothing operator ${ }^{1}$. The maximization in Eq.(12) is done numerically using Sequential Least Square Programming [23] with boundary contraints of finding the solution $\widehat{\phi}_{p}^{(\ell)}$ in the domain $-20^{\circ} \leq \widehat{\phi}_{p}^{0} \leq 20^{\circ}$. Furthermore, in order to simplify the maximization processus, we impose $\widehat{\phi}_{M}^{(\ell)}=\widehat{\phi}_{M}^{0}$, where $M$ is the master track (this operation should have no consequences on the tomographic analysis since only the interferometric information with respect to the master track are useful). The interest of the Phase Linking algorithm has been shown in details in [9] with real and synthetic data.

The ojective is then to retrieve the phase screens from the estimated linked phases $\left\{\phi_{p}^{(\ell)}\right\}_{p=1 . . P}$. For this step, we apply an iterative procedure similar to the one developped by Tebaldini et al. in [9], for which the joint estimation of phase screens and targets elevations is proposed.

\section{B. Estimation of trajectory errors}

For point, surfacic or volumetric targets at altitude $z_{t}$, the linked phases $\left\{\phi_{p, t}^{(\ell)}\right\}_{p=1 . . P}$ for this target can be expressed as the form of Eq.(11) [9]:

$$
\phi_{p, t}^{(\ell)}-\phi_{M, t}^{(\ell)}=k_{z}^{p, M} z_{t}+\alpha_{p}-\alpha_{M}
$$

with the subscript $M$ indicating the master track. A simple hypothesis is to assume that the phase screens only result from uncertainities in the antennas positions. Indeed, for airborne systems, the atmospheric perturbations are limited

\footnotetext{
${ }^{1}$ The smoothing operation of the initialisation phases was done by attenuating high frequencies in the $2 \mathrm{D}$ Fourier domain:

$$
f(a)=\arg \left(\mathrm{TF}^{-1}\left[\mathrm{TF}\left[e^{j a}\right] \times M\right]\right),
$$

where TF is the Fourier transform operator and $M$ is a mask such that:

$$
M= \begin{cases}M_{q} & \text { if }(u, v) \in D, \\ 0 & \text { else }\end{cases}
$$

where $(u, v)$ are the coordinates in the 2D Fourier plane, $D$ is a domain centered on 0 of size $d \times d$ with $d=25$ here, and $M_{q}$ is a normalized quadratic mask.
}

(no ionophere propagation). Moreover, for the specific P-band AfriSAR acquisitions, it appears that the ONERA Inertial Motion Unit (IMU) system located onboard was not accurate enough to detect distance variations between two trajectories smaller than the incident wavelength, which supports the idea of phase screens originating essentially from trajectory errors. Under this hypothesis, it can be shown that the phase screens $\alpha_{p}$ can be approximated as a function of $d Z_{p}$ and $d Y_{p}$ which represent respectively the position errors of the plateform $p$ in altitude and in the ground range direction for a fixed position in azimuth [9]:

$$
\alpha_{p} \simeq F\left(d Y_{p}, d Z_{p}, \theta\right)=\frac{4 \pi}{\lambda}\left(-d Y_{p} \sin \theta+d Z_{p} \cos \theta\right) .
$$

An estimator of $d Y_{p}, d Z_{p}$ and $z_{t}$ consists in finding the function $F\left(d \widehat{Y}_{p}, d \widehat{Z}_{p}, \theta\right)$ and the value $\widehat{z}_{t}$ that are the most representative of the linked phases difference of Eq.(17). For a fixed azimuth position in the image $p$, this step is done by selecting a number $T$ of pixels with different incident angles. The Double Localization iterative procedure described in details in [9] is then put in place. We differ from this method only by the initialisation step that we describe in the following.

For the chosen position in azimuth, we initialize the iterative algorithm of [9] by setting $\widehat{z}_{t}=0$ for $t=1$..T. This is a sensible hypothesis for the initialisation since phase calibration of the AfriSAR data has been done using TanDEM-X DEM, which is assumed to be close to the canopy surface. A circular criterion is then applied to estimate the initial position errors $d \widehat{Y}_{p}^{0}$ and $d \widehat{Z}_{p}^{0}$ :

$$
\left\{d \widehat{Y}_{p}^{0}, d \widehat{Z}_{p}^{0}\right\}=\operatorname{argmin}\left[\sum_{t} \arg \left(e^{j\left(F\left(d Y_{p}, d Z_{p}, \theta_{t}\right)-\widehat{\phi}_{p, t}^{\ell}\right)}\right)\right],
$$

where the linked phases $\widehat{\phi}_{p, t}^{\ell}$ and the angles $\theta_{t}$ for $t=1 . . T$ are calculated with an average window of size $33 \times 33$ centered on the pixels of interest.

From these initial estimated values of $d Y_{p}, d Z_{p}$ and $z_{t}$, we then procede as proposed in [9]. We compute the residual phases $\widehat{\phi}_{p, t}^{\ell}-F\left(d \widehat{Y}_{p}^{0}, d \widehat{Z}_{p}^{0}, \theta\right)$ for all the images to obtain an estimation $\widehat{z}_{t}$ for $t=1 . . T$ that is not necessarily null. We then use this estimated value to calculate the residual phases $\widehat{\phi}_{p, t}^{\ell}-\widehat{\phi}_{M, t}^{\ell}-k_{z}^{p, M} \widehat{z}_{t}$ and to update the values of $d \widehat{Y}_{p}$ and $d \widehat{Z}_{p}$, etc. As before, in order to simplify the process, the values of $d \widehat{Y}_{M}$ and $d \widehat{Z}_{M}$ for each iteration are fixed to their initial values $d \widehat{Y}_{M}^{0}$ and $d \widehat{Z}_{M}^{0}$ from Eq.(19).

As pointed out in [9], an ambiguity of half a wavelength in the direction of the incidence angle $\theta$ is present in the definition of $\alpha_{p}$ of Eq.(18), due to the $2 \pi$ phase ambiguity. Indeed, it is easy to see with Eq.(18) that a displacement on the sensor position error $\left(d Y_{p}, d Z_{p}\right)$ of $\delta Y_{p}=a \lambda / 2 \sin \theta$ and $\delta Z_{p}=a \lambda / 2 \cos \theta$, with $a$ an integer, leads to the same value of $F\left(d Y_{p}, d Z_{p}, \theta\right)$. To illustrate the consequences of this ambiguity on the estimation of $d Y_{p}$ and $d Z_{p}$, we introduce the function

$$
G(d Y, d Z)=\sum_{t=1}^{T}\left|\arg \left(e^{j B\left(d Y, d Z, d Y^{0}, d Z^{0}, \theta_{t}\right)}\right)\right|,
$$

with $B\left(d Y, d Z, d Y^{0}, d Z^{0}, \theta_{t}\right) \quad=\quad F\left(d Y, d Z, \theta_{t}\right) \quad-$ $F\left(d Y^{0}, d Z^{0}, \theta_{t}\right)$. The function $G(d Y, d Z)$ is represented 
in Figures 3(a) and 3(b) with $d Y^{0}=d Z^{0}=-0.2$, $d Y$ and $d Z$ in $[-1.0,1.0]$ for $T=2$ and $T=3$, with $\left[\theta_{1}, \theta_{2}, \theta_{3}\right]=\left[25^{\circ}, 55^{\circ}, 45^{\circ}\right]$. Every couple $\{d Y, d Z\}$ that leads to $G(d Y, d Z)=0$ is a valid solution. For $T=2$, it can be seen on Figure 3(a) that there exists an infinity of valid couples. For $T=3$, this ambiguity seems to be solved since there is only one global minimum reached for $d Y=d Z=-0.2$ (see the cut in logscale on Figure 3(c)) However the local minima stay close to zero: when estimatins the position errors in the presence of noise, it may be difficul to identify a single solution. The same conclusions stand for $T>3$.

Let's insist on the fact that this ambiguity has no consequence: on the value of the phase screens. It just incites one to take the estimation results of $d Y_{p}$ and $d Z_{p}$ with caution.

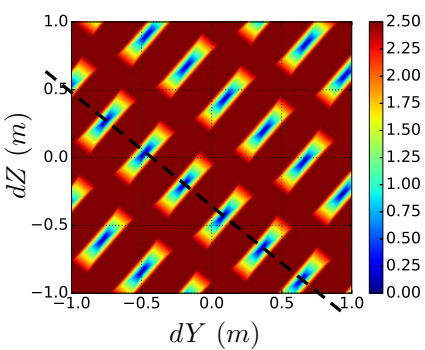

(a) $T=2$

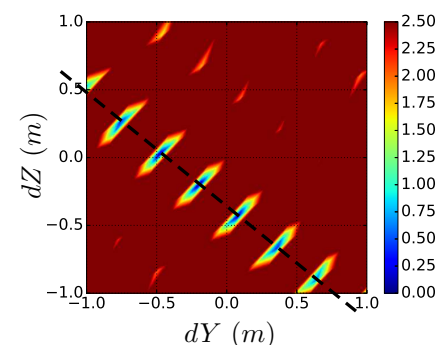

(b) $T=3$

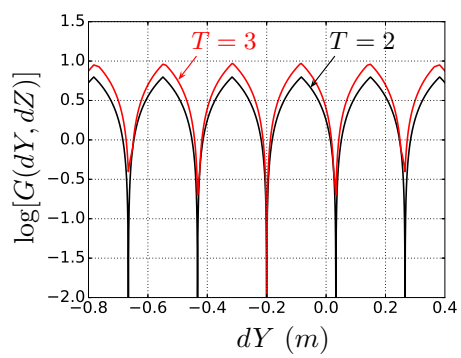

(c) Cut

Fig. 3. Function $G$ of Eq.(20) in the plane $(d Y, d Z)$ with $d Y^{0}=d Z^{0}=$ -0.2 for (a) $T=2$ and (b) $T=3$ with $\left[\theta_{1}, \theta_{2}, \theta_{3}\right]=\left[25^{\circ}, 55^{\circ}, 45^{\circ}\right]$. The values superior to 2.50 appear in dark red. (c) In logscale, cut in the direction represented by the dashed lines in (a) and (b).

This ambiguity also rises the problem of continuity in the estimations of $d Y_{p}$ and $d Z_{p}$ along the azimuth direction. To enforce continuity, we procede as proposed in [9] and initialize the optimization of Eq.(19) with the solution of the neighbor azimuth position. For more robustness, we also contraint the solution to be in a restraint domain around the initialization, considering that the antennas plateforms are slowly moving along the azimuth direction.

The estimations of $d Y_{p}$ and $d Z_{p}$ on La Lopé data are shown in Figure 4 for the 10 available images, using $T=7$ values of $\widehat{\phi}_{p, t}^{\ell}$ for the estimation of the $H V$ polarization channel and an initialization mid-azimuth. The results have been smoothed with a sliding windows of 400 pixels wide to attenuate the fluctuations. Position errors are estimated between $-60 \mathrm{~cm}$ and $+40 \mathrm{~cm}$ in the range direction, and between $-50 \mathrm{~cm}$ and $+50 \mathrm{~cm}$ in the altitude direction. However, we need to remember that, because of phase ambiguities, the $d Y_{p}$ and $d Z_{p}$ estimations are not unique.

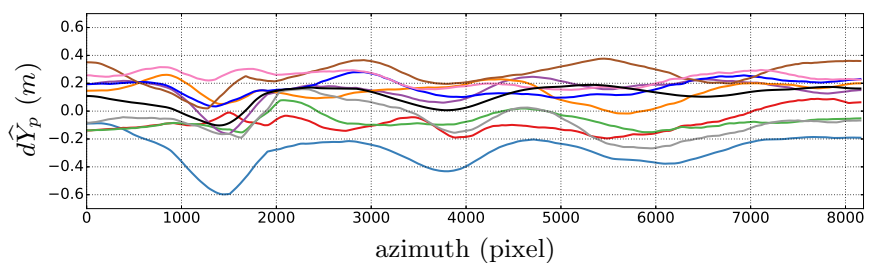

(a)

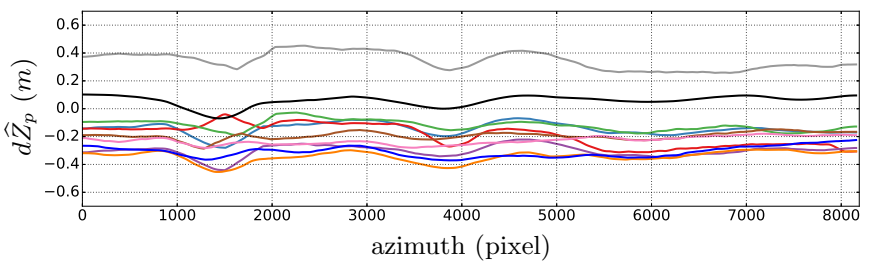

(b)

Fig. 4. Estimated trajectory errors $d \widehat{Y}_{p}$ and $d \widehat{Z}_{p}$ as a function of the azimuth position, obtained from Eq.(19) for $T=7$ values over La Lopé site for the 10 flights and after smoothing with a sliding window of 400 pixels large.

\section{Estimation of phase screens and correction of the data stack}

The final estimated phase screens are obtained from the antennas position errors $d \widehat{Y}_{p}$ and $d \widehat{Z}_{p}$ :

$$
\widehat{\alpha}_{p}=\frac{4 \pi}{\lambda}\left(-d \widehat{Y}_{p} \sin \theta+d \widehat{Z}_{p} \cos \theta\right) .
$$

The multibaseline data set is then processed by removing the phase screens from each track:

$$
s_{p}^{c o r r}=s_{p} e^{-j \widehat{\alpha}_{p}}, \text { for } p=1 . . P .
$$

The phase screens are supposed not to depend on the chosen polarization channel, only on the errors of plateforms trajectories. Then, for La Lopé, although $\widehat{\alpha}_{p}$ have been obtained from the $H V$ channel only, the same estimated phase screens are used for the correction of the other polarization channels. As an illustration, the estimated phase screens for the tracks 1,7 and 10 of the La Lopé multibaseline data set are represented on Figure 5.

\section{FIRST TOMOGRAPHIC ANALYSIS OVER LA LOPÉ FOR P-BAND ACQUISITIONS}

In this section, we analyze tomographic results obtained before and after applying the proposed correction procedure of section IV of the La Lopé P-band data. We focus on the ROIs identified in section II and on larger zones of the La Lopé site. The tomographic profiles are compared to both the SFL data and the LVIS data.

The tomographic profiles are compared to both the LIDAR data acquired by S. Saatchi (JPL) and by the NASA's LVIS instrument. We then focus in a second place on results obtained for larger zones of the La Lopé site.

\section{A. Impact of the phase screen correction}

Four particular ROIs presented in section II are analyzed in this paper: SAV2, COL1, COL3 and OKO2 (see table III for more details on the type and the number of pixels $N$ of the 


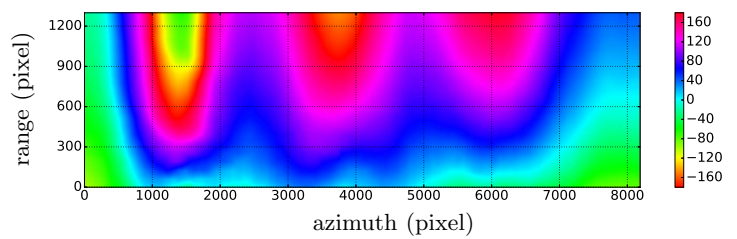

(a) Track 1

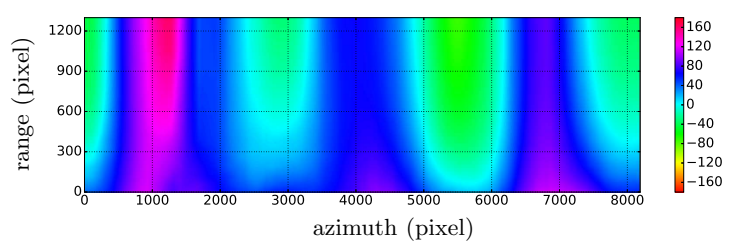

(b) Track 7

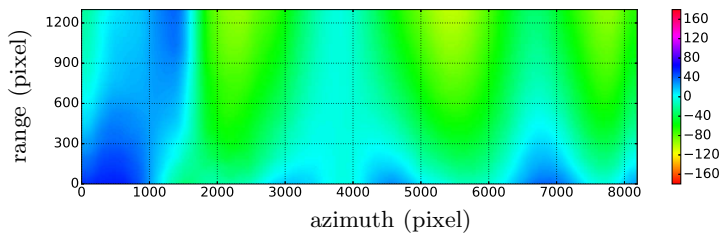

(c) Track 10

Fig. 5. Estimated phase screens $\widehat{\alpha}_{p}$ in degrees from Eq.(21) for La Lopé multibaseline P-band acquisitions for tracks 1,7 and $10(p=1,7,10)$.

ROIs). The Capon estimator of Eq.(7) is applied on the whole ROI areas.

To show the impact of the phase screen correction described in section IV, the vertical tomographic profiles $\widehat{S}_{C}(z)$ for the SAV2 region are shown before and after correction on Figure 6 for the $H V$ channel. The profiles have been normalized regarding to their maxima in the chosen vertical sampling. For the sake of representation, the mean TanDEM-X value over each of the ROIs is added to the vertical sampling of the Capon profile. In order to compare the tomographic profiles to the SFL data for each region, we superpose the mean ground SFL elevation (i.e. the mean DTM) on the whole region in black continuous line and the mean canopy SFL elevation in green dashed line, which is simply the sum of the mean DTM and the mean CHM on the whole region. The histograms of ground elevation and canopy elevation are also shown on the tomographic profiles.

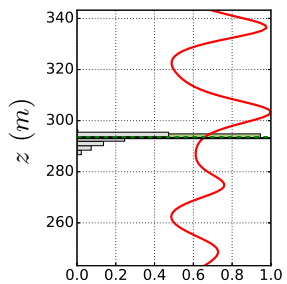

(a) Before correction

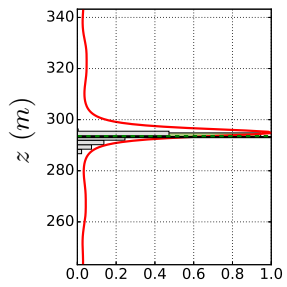

(b) After correction
Fig. 6. In red, normalized tomographic profiles $\widehat{S}_{C}(z)$ from the Capon estimator applied on the ROI SAV2 of Table III for the $H V$ polarimetric channel. The histograms of SFL ground and canopy elevations are represented respectively in grey and in green. The means of the histograms are represented respectively in continuous black line and dashed green line. In the case of SAV2, the vegetation is very low and the lines are pratically superposed. (a): Profile without any phase screen correction. (b): Profile with the phase screen correction described in the section IV.
Comparing the SAV2 Capon profiles of Figure 6, it can be seen that the phase screen correction procedure described in section IV significantly improves the vertical profiles of this ROI. Indeed, the correction procedure leads to a better focusing of the principal lobe when compared to the SFL data, with a difference of less than $1 \mathrm{~m}$ between the position of the peak of the principal lobe and the mean DTM. The phase screen correction also provides a strong reduction of the side lobes power, moving from more than $70 \%$ of the principal lobe power to less than $10 \%$ for the SAV2 region. After correction, the width of the principal lobe for SAV2 (which located nearrange in the SLC images) is of about $4.1 \mathrm{~m}$ at $-3 \mathrm{~dB}$.

The results after correction for the other ROIs for the $H V$ and the $H H$ channels are presented respectively on Figure 7 and on Figure 8. It appears on Figure 7 and Figure 8 that the side lobes powers after correction for all the other analyzed ROIs represent less than $20 \%$ of the most energetic principal lobe power, with a difference between the peaks of the principal lobes (ground and vegetation) and the corresponding mean LIDAR data of less than $5 \mathrm{~m}$.

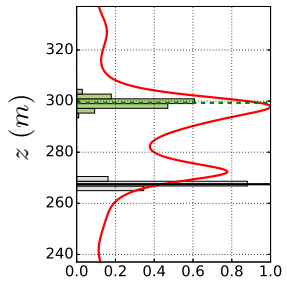

(a) $\mathrm{OKO} 2$

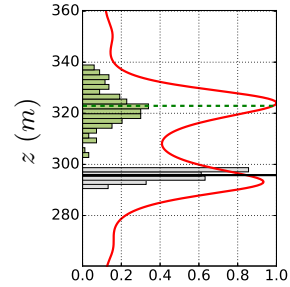

(b) COL1

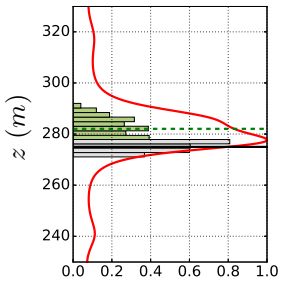

(c) COL3
Fig. 7. Idem as in Figure 6 but after phase screen correction for the ROIs (a) OKO2, (b) COL1, and (c) COL3 of Table III for the $H V$ channel

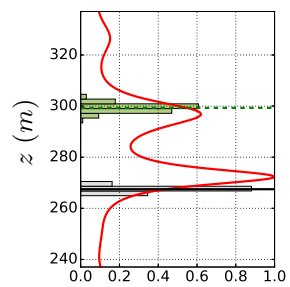

(a) $\mathrm{OKO} 2$

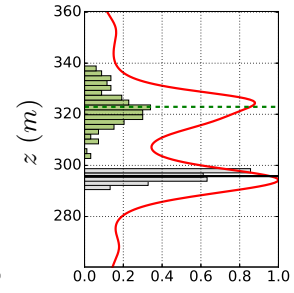

(b) COL1

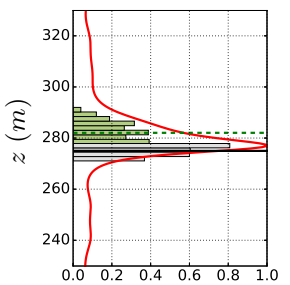

(c) COL3
Fig. 8. Idem as in Figure 7 but for the $H H$ channel.

\section{B. Analysis of the tomographic results on ROIs}

When observing the Capon profiles after phase screen correction for the ROIs OKO2, COL1 and COL3 on Figures 7 and 8 , it is worth noticing that the width of the principal lobes is correlated to the distribution of the SFL data in the corresponding region, opening the perspective of estimation of the vertical structure of the La Lopé forest with tomographic analysis.

Comparing the $H V$ results (Figure 7) and the $H H$ results (Figure 8), it can also be seen that the ground contribution is stronger in comparison to the volume contribution for the $H H$ channel than for the $H V$ channel for the analyzed ROIs. This behavior was expected for P-band imaging, for which the 
relative contribution of the canopy with respect to the ground is larger for $H V$ than for $H H$.

\section{Comparison with LVIS profiles}

The vegetation profiles of 3 of the ROIs of Table III have been obtained from the LVIS Level1B data presented in section II. Although not presented here, the analysis has also been done using the Level2 data, which leads to similar conclusions. The normalized mean LVIS profiles over each ROI are presented in blue on Figure 9. For sake of representation, the mean TanDEM-X value on the corresponding ROI has been added to the vertical sampling of the LVIS profiles. As before, the histograms of the SFL data for ground elevation (DTM) and for canopy elevation (CHM plus mean DTM) are shown respectively in grey and green. The corresponding Capon profiles for the $H V$ channel are also shown in red.

Although the shapes of the vertical profiles may differ, the mean LVIS profiles show similitudes with the tomographic Capon profiles after phase screen correction. In particular, the vegetation lobe for both LVIS and Capon profiles is narrower for OKO2 than for COL1 (see Figures 9(a) and 9(b)), which is correlated to the width of the SFL canopy elevation distribution for these two regions.

The difference of shapes between the LVIS and the Capon profiles may originate for example from a difference in penetration into the vegetation or a difference of profile resolution. Indeed, for the ROIs OKO2 and COL1 of Figures 9(a) and (b), it clearly appears that the ground lobe is higher than $75 \%$ of the vegetation lobe for the $H V$ Capon profiles, while it is less marked for the LVIS profiles. This could be the consequence of a weaker penetration of the LVIS system through the vegetation volume compared to the P-band SAR imaging. It is also worth noticing on Figure 9(b) that the COL1 LVIS profile presents a secondary vegetation lobe above the principal one that seems to correspond well to the canopy elevation distribution. This lobe however does not appear on the Capon profile, which could possibly be explained by the lower resolution of the Capon estimator. This lack of resolution may also explain the shape of the COL3 Capon profile compared to the LVIS profile of Figure 9(c). Indeed, the Capon estimator cannot separate the ground and the vegetation contributions for this young colonizing forest, which translates into a large single principal lobe, whereas the LVIS profile shows sharps ground and vegetation lobes that follow the canopy and terrain elevation distributions.

As a conclusion on the comparison, the LVIS and the Capon resuls show great similitaries. Although, tomography SAR in P-band with Capon estimator may fail to produce vertical profiles with a resolution as good as the LVIS imaging system, it always reveals a stronger ground contribution on the studied ROIs.

\section{Analysis on cuts in the azimuth and in the range directions}

In this paragraph, we present estimated tomographic profiles for a cut of the La Lopé site in the azimuth or in the range direction. The Capon analysis have been applied on sliding windows of $33 \times 33$ pixels $(N=1089$ pixels total) for each

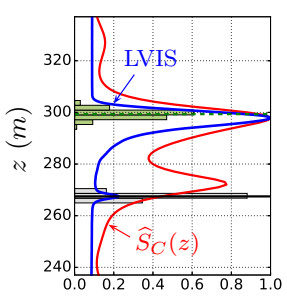

(a) $\mathrm{OKO} 2$

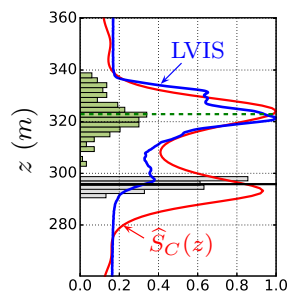

(b) COL1

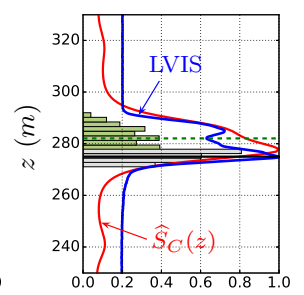

(c) COL3
Fig. 9. In blue, mean normalized LVIS profiles for 3 of the ROIs of Table III. In red, normalized Capon profiles $\widehat{S}_{C}(z)$ for the $H V$ channel. The histograms of SFL ground and canopy elevations are represented respectively in grey and in green. The means of the histograms are represented respectively in continuous black line and dashed green line. (a): OKO2. (b): COL1. (c): COL3.

position in azimuth and range.

Figure 10 presents the tomographic profiles $\widehat{S}_{C}(z)$ in the azimuth direction for a mid-range section cut (sliding window centered on the pixel number 799). On Figure 10(a) the tomographic profile is presented before any phase screen correction for the $H V$ channel. The results after correction are shown on Figure 10(b) for the $H V$ channel and on Figure 10(c) for the $H H$ channel. As before, we superpose the mean ground LIDAR elevation and the mean canopy LIDAR elevation that have been calculated on the sliding window. The normalization of the profiles has been done for each position in azimuth. Similar conclusion as the ones for the ROIs analysis can be drawn from the observation of the estimated profiles. Indeed, the improvement brought by the phase screen correction for this region is well visible, providing side lobes attenuation and better correlation with the LIDAR data. Moreover, the ground contribution is higher in the $H H$ channel than in the $H V$ channel again. It can also be noticed that, for the Capon estimator, the power of the vertical profile seems to be concentrated essentially on the ground and on the top of the canopy, with a clear diminution of power between these two layers, when they are sufficiently spaced.

The same analysis is done in the range direction for a fixed azimuth position (sliding window centered on the pixel number 2816). The Capon profile for the $H H$ channel is shown on Figure 11 for the corrected data only. On this profile, the effect of the slopes on the backscattered power can be noticed. When the slope faces the radar (for example from pixels number 120 to 180 in range direction on Figure 11 ), the ground contribution comparing to the canopy one is dominating. However, when the slopes is away from the radar antennas (for example from pixels number 200 to 270 in range direction), the ground contribution fades significantly, which rises the question of ground detection and elevation estimation from tomographic analysis in the presence of back slopes.

It can also be noticed on Figures 10 and 11 that, even if the SFL data and the corrected tomographic profiles seem to have generally a good correspondance, the mean SFL elevations can sometimes notably differ from the peaks positions of the Capon profiles (for exemple between pixels 5400 and 5600 in azimuth direction on Figure 10(c)). This could be linked to the SFL data that do not describe the exact same scene due to the difference in forest penetration or to other uncompensated 


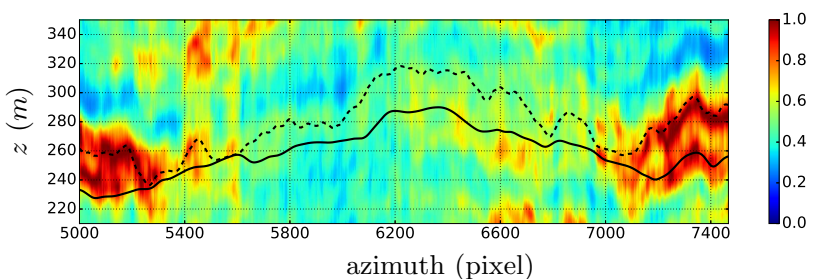

(a) $H V$ before correction

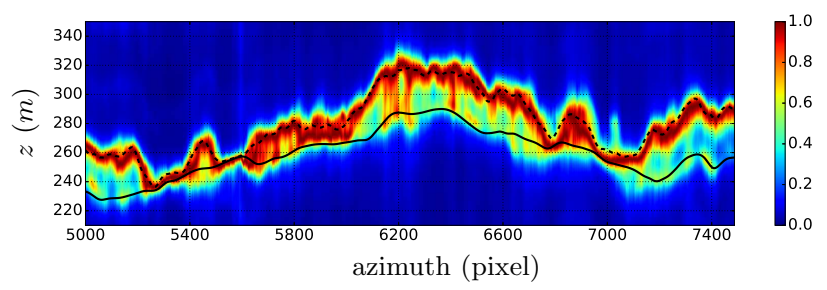

(b) $H V$ after correction

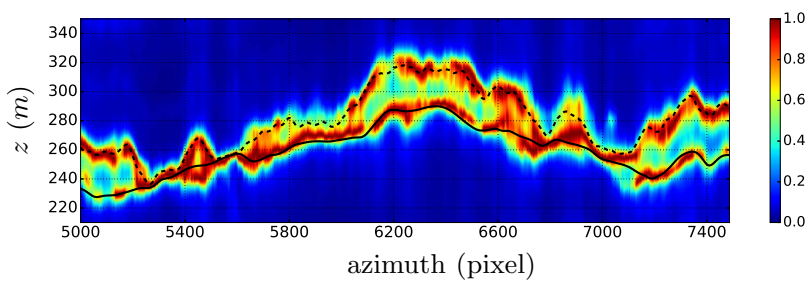

(c) $H H$ after correction

Fig. 10. Tomographic profile $\widehat{S}_{C}(z)$ from the Capon estimator in the azimuth direction for a fixed range position in La Lopé. The analysis is done with a $33 \times 33$ pixels sliding window. The continuous black line and the dashed black line indicate respectively mean ground and mean canopy SFL elevation on the sliding window. (a): For the $H V$ channel without any phase screen correction. (b): For the $H V$ channel with the phase screen correction described in the section IV. (c): Idem as in (b) but for the $H H$ channel.

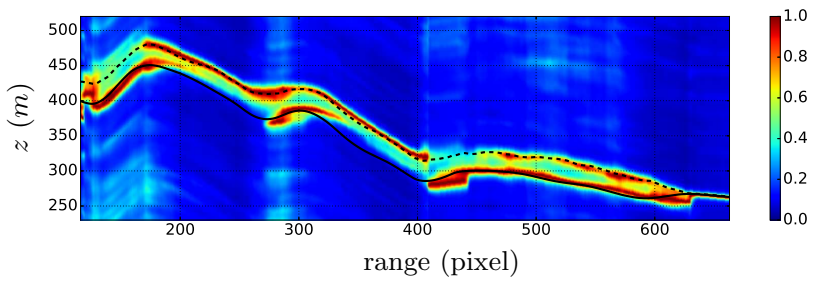

Fig. 11. Idem as in Figure 10 but in the range direction for a fixed azimuth position in La Lopé for the $H H$ channel with the phase screen correction described in section IV

effects. An interesting perspective to this work is to analyze precisely this type of situations.

\section{CONCLUSION}

The AfriSAR campaign was conducted between 2015 and 2016 in Gabon (Africa) by ONERA and DLR. On ONERA side, the SETHI airborne system has imaged four different sites over Gabon during July 2015. The main objective of this campaign was to collect data in tropical context in order to support the development of the ESA BIOMASS future mission of global observation of Earth biomass with spaceborne systems. On ONERA side, the AfriSAR campaign was also part of a study of the potential of SAR imaging to access undercanopy terrain caracteristics for TOTAL geomorphology analyses.
Tomographic SAR analysis is a promising technique for the estimation of forest structure and undercanopy topography, and has been applied on the P-band multibaseline data collected with the SETHI system over La Lopé National Park. It has however been shown that this analysis is difficult to conduct without a preliminary correction of the residual phases (phase screens) that are perturbing the acquisitions and creating artefacts on the tomographic profiles. A phase screen correction method has been proposed in this paper and applied on the $\mathrm{La}$ Lopé data. The described correction procedure is based on the Double Localization iterative algorithm proposed by Tebaldini et al. [9], with Phase Linking algorithm [22], [9] and under the hypothesis of phase screens originating only from antennas position errors for these airborne acquisitions.

The tomographic Capon profiles after correction on different regions of the La Lopé site show generally a good correspondance with the Small Footprint LIDAR data collected by JPL and the data from the NASA's Land Vegetation Ice Sensor, with side lobes power under $20 \%$ of the most energetic principal lobe power. In particular, on several regions of interest, it has been seen that the width of the principal lobes of the Capon profile appears to be correlated with the distribution of SFL ground and canopy elevations within the zone. The analysis seems to be more difficult for hilly topographies, because of the partial loss of the ground contribution in comparison to the volume due to large back slopes.

The most immediate perspective to this work is to pursue this first tomographic analysis by developping an estimator of the vegetation height and ground elevation from the Capon profiles, and by studying its performance and robustness. The caracterisation of the obtained Digital Elevation Model would then be an interesting analysis to do and to compare to other existing DEM such as the LIDAR one.

Moreover, in recent studies [7], [8], SAR tomography at P-band has been shown to lead to accurate estimations of aboveground biomass (AGB) for tropical forests in French Guiana. In further work, it will be interesting to extend this study to the data collected at La Lopé site.

Another interesting perspective is the use of the corrected multibaseline data in PolInSAR context [24] and to compare the results with the one obtained with tomography. Testing the phase screen correction method and analyzing the tomographic results from the multibaseline data acquired over the other Gabon forests sites is also an on-going work.

The evolution of the performance for satellite configuration is also an important question for the BIOMASS mission. In this context, the impact of the resolution of the images on the quality of the tomographic profile is an interesting point to study. Furthermore, for spaceborne acquisitions, the origins of the phase screens will probably be different than for airborne acquisitions: position errors for satellite plateforms are indeed expected to be negligible, while perturbations causing by the propagation in the atmosphere cannot be ignored anymore. The evolution of the correction procedure in this context is then a crucial analysis to carry on. 


\section{ACKNOWLEDGMENT}

The ONERA AfriSAR campaign was supported by ESA, CNES and TOTAL. The authors want to thank the AfriSAR ground measurements team from CESBIO and UCL, and the ONERA airborne radar team who was responsible of the radar acquisitions. The authors are also grateful to the NASA for their collaboration during the campaign and the collect of the LIDAR data. The authors would also like to express their deep gratitude to the local Gabon agencies AGEOS and ANPN and to DLR, the other SAR operator with whom the overall campaign was defined.

\section{REFERENCES}

[1] T. Le Toan, S. Quegan, M. W. J. Davidson, H. Balzter, Ph. Paillou, K. P. Papathanassiou, S. Plummer, F. Rocca, S. Saatchi, H. Shugart and L. Ulander, The BIOMASS mission: Mapping global forest biomass to better understand the terrestrial carbon cycle," Remote Sens. Environ., vol. 115, no. 11, pp. 2850-2860, Nov. 2011.

[2] P. C. Dubois-Fernandez, X. Dupuis, P. Capdessus and R. Baqué, "Preliminary results of the AfriSAR campaign," in Proc. EUSAR 2016: 11th European Conference on Synthetic Aperture Radar, pp. 1-3, Jun. 2016.

[3] A. Reigber and A. Moreira, "First Demonstration of Airborne SAR Tomography Using Multibaseline L-Band Data," IEEE Trans. Geosci. Remote Sens., vol. 38, no. 5, pp. 2142-2152, Sep. 2000.

[4] F. Gini, F. Lombardini and M. Montanari, "Layover Solution in Multibaseline SAR Interferometry," IEEE Trans. Aerosp. Electron. Syst., vol. 38, no. 4, pp. 1344-1356, Oct. 2002.

[5] S. Tebaldini, "Algebric Synthesis of Forest Scenarios From Multibaseline PolInSAR Data," IEEE Trans. Geosci. Remote Sens., vol. 47, no. 12, pp. 4132-4142, Dec. 2009.

[6] Y. Huang, L. Ferro-Famil and A. Riegber, "Under-Foliage Object Imaging Using SAR Tomography and Polarimetric Spectral Estimators," IEEE Trans. Geosci. Remote Sens., vol. 50, no. 6, pp. 2213-2225, Jun. 2012.

[7] D. Ho Tong Minh, T. Le Toan, F. Rocca, S. Tebaldini, M. Mariotti d'Alessandro and L. Villard, "Relating P-Band Synthetic Aperture Radar Tomography to tropical Forest Biomass," IEEE Trans. Geosci. Remote Sens., vol. 52, no. 2, pp. 967-979, Feb. 2014.

[8] D. Ho Tong Minh, T. Le Toan, F. Rocca, S. Tebaldini, L. Villard, M.|Réjou-Méchain, O. L. Phillips, T. R. Feldpausch, P. C. DuboisFernandez, K. Scipal and J. Chave, "SAR tomography for the retrieval of forest biomass and height: Cross-validation at two tropical forests sites in French Guiana," Remote Sens. Environ., vol. 175, no. Supplement C, pp. 138-147, Mar. 2016.

[9] S. Tebaldini, F. Rocca, M. Mariotti d'Alessandro and L. Ferro-Famil, "Phase Calibration of Airborne Tomographic SAR Data via Phase Center Double Localization," IEEE Trans. Geosci. Remote Sens., vol. 54, no. 3, pp. 1775-1792, Mar. 2016.

[10] P. Axelsson, "DEM generation from laser data using adaptative TIN models," International Archive of Photogrammetry and Remote Sensing, vol. 33, no. (B4/1; PART 4), pp. 111-118, 2000.

[11] J. B. Blair, D. L. Rabine and M. A. Hofton, "The Laser Vegetation Imaging Sensor: a medium-altitude, digitalisation-only, airborne, laser altimeter for mapping vegetation and topography," ISPRS J. Photogramm. Remote Sens., vol. 54, no. 2, pp. 115-122, Jul. 1999.

[12] https://lvis.gsfc.nasa.gov

[13] R. Bamler and Ph. Hartl, "Synthetic aperture radar interferometry," Inverse Problems, vol. 14, no. 4, pp. R1, 1998.

[14] J. W. Goodman, "Statistical Optics," John Wiley and Sons, Inc., 1985.

[15] S. Tebaldini and A. Monti Guarnieri, "On the Role of Phase Stability in SAR Multibaseline Applications," IEEE Trans. Geosci. Remote Sens., vol. 48, no. 7, pp. 2953-2966, Jul. 2010.

[16] S. Tebaldini, "Multibaseline SAR Imaging: Models and Algorithms," Ph.D. dissertation, Dept. Electron. Inf., Politecnico di Milano, Italy, 2009.

[17] P. Stoica and R. Moses, Spectral Analysis of Signals, Prentice Hall, 2005.

[18] G. Fornara, G. Franceschetti and S. Perna, "Motion compensation errors: effects on the accuracy of airborne SAR images," IEEE Trans. Aerosp. Electron. Syst., vol. 41, no. 4, pp. 1338-1352, Oct. 2005.
[19] H. A. Zebker and P. A. Rosen, "Atmospheric artifacts in interferometric SAR surface deformation and topographic maps," J. Geophys. Res., Jun. 1996.

[20] A. Ferretti, C. Prati and F. Rocca, "Permanent Scatterers in SAR Interferometry," IEEE Trans. Geosci. Remote Sens., vol. 39, no. 1, pp. 820, Jan. 2001.

[21] S. Tebaldini and F. Rocca, "On the Impact of Propagation Disturbances on SAR Tomography: Analysis and Compensation," in Proc. IEEE Radar Conf., pp. 1-6, 2009.

[22] A. Monti Guarnieri and S. Tebaldini, "On the Explotation of Target Statistics for SAR Interferometry Applications," IEEE Trans. Geosci. Remote Sens., vol. 46, no. 11, pp. 3436-3443, Nov. 2008.

[23] D. Kraft, "A software package for sequential quadratic programming," Tech. Rep. DFVLR-FB 88-28, DLR German Aerospace Center - Institute for Flight Mechanics, Koln, Germany, 1988.

[24] K. Papathanassiou and S. R. Cloude, "Single-baseline polarimetric SAR interferometry," IEEE Trans. Geosci. Remote Sens., vol. 39, no. 11, pp. 2352-2363, Nov. 2001. 